\title{
Insulin upregulates the expression of zinc finger and BTB domain-containing 7A in HepG2 cells
}

\author{
JING TIAN $^{1,2}$ and YUYANG JIANG ${ }^{2,3}$ \\ ${ }^{1}$ School of Life Sciences, Tsinghua University, Beijing 100084; ${ }^{2}$ The Ministry-Province Jointly Constructed Base \\ for State Key Laboratory, Shenzhen Key Laboratory of Chemical Biology, The Graduate School at Shenzhen, \\ Tsinghua University, Shenzhen 518055; ${ }^{3}$ Department of Pharmacology and Pharmaceutical Sciences, \\ School of Medicine, Tsinghua University, Beijing 100084, P.R. China
}

Received May 16, 2012; Accepted September 10, 2012

DOI: $10.3892 / \mathrm{mmr} .2012 .1113$

\begin{abstract}
Zinc finger and BTB domain-containing 7A (Zbtb7A) is a proto-oncogene overexpressed in numerous cancers. In this study, we explored the mechanism of insulin-induced Zbtb7A expression. Real-time PCR and western blotting were used to detect Zbtb7A expression. Zbtb7A promoter activity was monitored by Luciferase reporter assay. It was shown that insulin elevates the mRNA and protein levels of the Zbtb7A gene in HepG2 cells. Using chemical inhibitors of insulin downstream pathways, we demonstrated that the insulin-induced Zbtb7A gene expression was completely blocked by LY294002, a PI3K/AKT inhibitor, and partially attenuated by the MAPK inhibitor PD98059. Transfection of HepG2 cells with a $1 \mathrm{~kb}$ Zbtb7A promoter-luciferase reporter construct revealed a dose-dependent activation of the Zbtb7A promoter by insulin, while mutation of the Sp1 binding site within the Zbtb7A promoter resulted in the failure of insulin-induced promoter activation, suggesting that insulin increases Zbtb7A expression through transcriptional regulation mediated by $\mathrm{Sp} 1$ in HepG2 cells.
\end{abstract}

\section{Introduction}

Human zinc finger and BTB domain-containing 7A (Zbtb7A), also known as POK erythroid myeloid ontogenic factor (pokemon) (1), factor that binds to IST, the HIV-1 inducer of short transcripts (FBI-1) (2), leukemia/lymphoma-related factor mouse homolog (LRF) (3) and osteoclast-derived zinc finger rat homolog (OCZF) (4), was originally identified as a cellular factor that binds specifically to the human immunodeficiency

Correspondence to: Professor Yuyang Jiang, The MinistryProvince Jointly Constructed Base for State Key Laboratory, Shenzhen Key Laboratory of Chemical Biology, The Graduate School at Shenzhen, Tsinghua University, 2279 Lishui Road, Shenzhen 518055, P.R. China

E-mail: jiangyy@sz.tsinghua.edu.cn

Key words: zinc finger and BTB domain-containing 7A, proto-oncogene, insulin, signaling pathway virus type 1 promoter element (5). Zbtb7A belongs to the POK protein family (6) and plays critical roles in differentiation (7), oncogenesis $(1,8)$ and adipogenesis $(9)$. The overexpression of Zbtb7A is observed in various human cancers (1). Zbtb7A suppresses the p14ARF, Rb tumor suppressor gene $(1,10)$, p21CIP1 (11), E2F4 and cyclin A (9), indicating that Zbtb7A may be a molecular target for cancer therapy.

Many Zbtb7A downstream targets have been identified, but little is known about the mitogens that regulate the expression of Zbtb7A. Roh et al reported that Zbtb7A activity was regulated by sumoylation (12). In addition, we have demonstrated previously that $\mathrm{Sp1}$, a well-known effector of insulin in cancer cells (13), elevates Zbtb7A expression by directly binding to its promoter (14). Based on this study, we hypothesized that there is a correlation between insulin and Zbtb7A expression.

Apart from its role in the regulation of glucose uptake, insulin also acts as a mitogen and differentiation factor in a variety of cells and tissues (15-18). As a mitogen, insulin regulates the transcription of $>100$ genes $(19,20)$ and promotes cell growth $(21,22)$. The actions of insulin are initiated through the activation of the insulin receptor, which is a receptor tyrosine kinase, and downstream serine/threonine kinase-signaling pathways including PI 3-kinase (PI3K/AKT) and Ras-MAPK cascades $(23,24)$. Boyd reported that the bioavailability of insulin contributes to tumor development (25). It has also been reported that insulin is able to stimulate the expression of the oncogene PTTG (26). In the current study, we demonstrate that the mRNA and protein levels of Zbtb7A are significantly upregulated by insulin via the PI3K/AKT pathway and the transcription factor $\mathrm{Spl}$.

\section{Materials and methods}

Materials. Insulin and polyclonal anti-Zbtb7A were obtained from Sigma (St. Louis, MO, USA). Goat anti-rabbit IgG horseradish peroxidase (HRP)-linked antibody, goat anti-mouse HRP-linked antibody, the PI3K/AKT inhibitor LY294002 and the MAPK inhibitor PD98059 were purchased from Beyotime Biotech (Jiangsu, China). The nylon membrane Hybond-N was obtained from Pall Corporation (Port Washington, NY, USA). The TRIzol reagent was purchased from Invitrogen Life Technologies (Carslbad, CA, USA). 
Cell culture. HepG2 cells were cultured in Dullbecco's modified Eagle's medium (DMEM) with 10\% fetal bovine serum (FBS), $100 \mathrm{U} / \mathrm{ml}$ penicillin and $100 \mu \mathrm{g} / \mathrm{ml}$ streptomycin in humidified air containing $5 \% \mathrm{CO}_{2}$ at $37^{\circ} \mathrm{C}$. Cells were trypsinized and seeded in 6-well plates and grown to 50-70\% confluence. They were then stimulated with various reagents as described in the figure legends.

RNA extraction and real-time PCR analysis. The total RNA of the HepG2 cells was isolated using TRIzol reagent. Reverse transcription was performed using a RT-PCR kit according to the instructions of the manufacturer (Toyobo, Osaka, Japan). The quantification of the Zbtb7A mRNA $(1 \mu \mathrm{g})$ was performed using an ABI 7500 real-time PCR system with the Zbtb7A specific primers: 5'-GAAGCCCTACGAGTGCAACATC-3' (forward) and 5'-GTGGTTCTTCAGGTCGTAGTTGTG-3' (reverse). GAPDH served as the internal control and was amplified with the following primers: 5'-GGTGGTCTCCTCT GACTTCAACA-3' (forward) and 5'-GTTGCTGTAGCCAAA TTCGTTGT-3' (reverse). The volume of real-time RT-PCR system was $20 \mu \mathrm{l}$, containing: $10 \mu \mathrm{l} \mathrm{SYBR-buffer,} 0.4 \mu \mathrm{l}$ forward primer $(10 \mu \mathrm{mol} / \mathrm{l}), 0.4 \mu \mathrm{l}$ reverse primer $(10 \mu \mathrm{mol} / \mathrm{l})$, $0.4 \mu 1$ SYBR-Green II dye, $1 \mu 1 \mathrm{cDNA}$ and $7.8 \mu 1$ water. Thermal cycling conditions were denaturation at $95^{\circ} \mathrm{C}$ for $10 \mathrm{sec}$, and then $5 \mathrm{sec}$ denaturation at $95^{\circ} \mathrm{C}, 34 \mathrm{sec}$ annealing at $60^{\circ} \mathrm{C}$ for 40 cycles, followed by a dissociation stage $\left(95^{\circ} \mathrm{C}\right.$ for $15 \mathrm{sec}, 60^{\circ} \mathrm{C}$ for $1 \mathrm{~min}$ and $95^{\circ} \mathrm{C}$ for $15 \mathrm{sec}$ ) to monitor the specificity of the primers. All samples were run in triplicate.

Site directed mutagenesis. The $\mathrm{Sp} 1$ recognition site of the Zbtb7A promoter was GGGCGG, -641-636 bp relative to the transcription start site. The mutation of this site was obtained by preparing the mutated promoter Sp1M, using mutated primers within GC-boxes. The plasmids with mutation were generated using a site-directed mutagenesis system (Promega, Madison, WI, USA) according to the instructions provided by the manufacturer and a pLuc1000 construct was used as a template. The oligonucleotide used for mutagenesis (mutations indicated with inclined form and bold letters) is: 5'-AATGATCCAA $\boldsymbol{A} \boldsymbol{A} \boldsymbol{A} \boldsymbol{A} \boldsymbol{A} \boldsymbol{A C T G C C T C C C A A G - 3 ' . ~ D N A ~}$ sequencing was performed to confirm that the sequences of the PCR products were correct as compared with the Zbtb7A promoter published in the Human Genome database.

Plasmid transfection and luciferase assay. Human pGL4.10-Zbtb7A promoter pLuc1000 (1000 bp, wild-type) or mutated promoter Sp1M was used for transient transfection. Cells were plated in 24-well plates. After culturing for $18 \mathrm{~h}$, the cells were transfected with Lipofectamine 2000 according to the instructions of the manufacturer (Invitrogen Life Technologies). Wild-type or mutated Zbtb7A promoter-luciferase construct ( $1 \mu \mathrm{g} /$ well) was used and $30 \mathrm{ng} / \mathrm{well}$ of pRLTK plasmid was co-transfected as a control reporter plasmid. Six hours after transfection, the cells were washed with PBS twice, cultured in DMEM containing 10\% FBS for $24 \mathrm{~h}$ and then treated with the indicated concentrations of insulin for $24 \mathrm{~h}$.

Cells were harvested by lysis buffer (Promega). Cell lysates $(10 \mu 1)$ were analyzed to determine the promoter activity. Three independent transfection experiments were carried out for each assay.
Western blot analysis. Following treatment with the indicated reagents, the cells were lysed with RIPA buffer containing $10 \mathrm{mM} \mathrm{KCl}, 0.1 \%$ Nonidet P-40, $10 \mathrm{mM}$ HEPES, pH 7.9, $1 \mathrm{mM}$ ethylenediamine tetraacetic acid (EDTA), $1 \mathrm{mM}$ phenylmethylsulfonyl fluoride (PMSF), $0.5 \mathrm{mM} \mathrm{Na}_{3} \mathrm{VO}_{4}$ and $1 \mathrm{mM}$ dithiothreitol (DTT). The protein concentration was determined by Bradford protein assay. Equal amounts of each protein sample $(30 \mu \mathrm{g})$ were resolved by SDS-PAGE and transferred to a nitrocellulose membrane. Blots were incubated in $5 \%$ non-fat milk at room temperature for $1 \mathrm{~h}$, washed three times with $0.5 \%$ Tween-20 Tris-buffered saline (TBST) and probed with a suitable primary antibody at room temperature for $2 \mathrm{~h}$. The membrane was washed and incubated with secondary antibody for $1 \mathrm{~h}$ at room temperature and detected using Quantity One software (Bio-Rad Laboratories, Hercules, CA, USA). $\beta$-actin was used to monitor the equal loading of each sample.

Statistical analysis. Statistical analysis was performed using ANOVA. Values of ${ }^{*} \mathrm{P}<0.05,{ }^{* *} \mathrm{P}<0.01$ and ${ }^{* * * *} \mathrm{P}<0.001$ were considered to indicate statistically significant differences.

\section{Results}

Insulin regulates $Z$ Zbtb7A expression in a dose-dependent manner. To determine whether insulin enhances Zbtb7A expression, HepG2 cells were treated with various concentrations of insulin or left untreated for $24 \mathrm{~h}$. The Zbtb7A mRNA and protein levels were analyzed by real-time PCR and western blot analysis, respectively. As shown in Fig. 1, insulin activated Zbtb7A expression in a dose-dependent manner. Compared with the control group, when the cells treated with 10,50 and $100 \mathrm{nM}$ insulin, the Zbtb7A mRNA levels were increased $1.21-, 1.45-(\mathrm{P}<0.05)$ and 2.17 -fold $(\mathrm{P}<0.01)$, respectively; similarly, the Zbtb7A protein levels were increased 1.47-, 1.49- and 2.09-fold (all $\mathrm{P}<0.05$ ), respectively (Fig. 1B and C).

Insulin stimulates Zbtb7A expression in a time-dependent manner. We then sought to discover the time-dependent effects of insulin on Zbtb7A expression. HepG2 cells treated with $100 \mathrm{nM}$ insulin were harvested at different time-points. It was found that the insulin-induced upregulation of Zbtb7A mRNA levels was time-dependent (Fig. 2A). The expression levels of Zbtb7A in the cells treated with $100 \mathrm{nM}$ insulin for 6,12 and $24 \mathrm{~h}$ were increased 1.38- $(\mathrm{P}<0.01), 2.01-(\mathrm{P}<0.05)$ and 2.51-fold $(\mathrm{P}<0.05)$, respectively, compared with untreated cells. In addition, a maximum 2.09-fold $(\mathrm{P}<0.001)$ increase in Zbtb7A protein levels was observed $24 \mathrm{~h}$ after insulin treatment, but no marked changes in Zbtb7A protein levels were detected at 6 and $12 \mathrm{~h}$ (Fig. 2B and C). These results suggest that insulin upregulates the mRNA and protein levels of Zbtb7A in HepG2 cells in a time-dependent manner.

Insulin-induced upregulation of Zbtb7A expression is mediated by the PI3K/AKT pathway. Insulin mediates target proteins through two major pathways: PI3K/AKT and MAPK. We used specific inhibitors of the two pathways to identify which of these pathways are involved in the insulin-induced Zbtb7A expression. HepG2 cells were pre-treated with LY294002 (a PI3K inhibitor) or PD98059 (a MAPK inhibitor) 
A

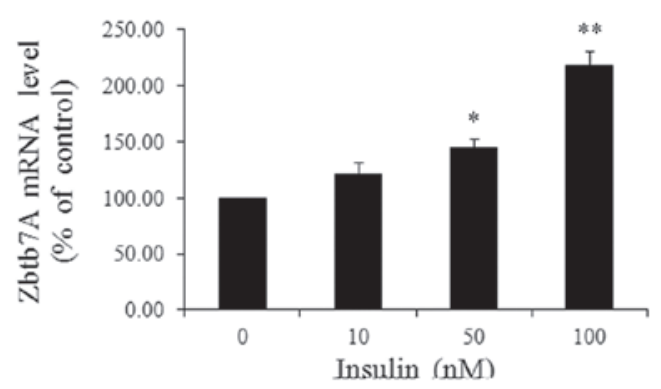

B

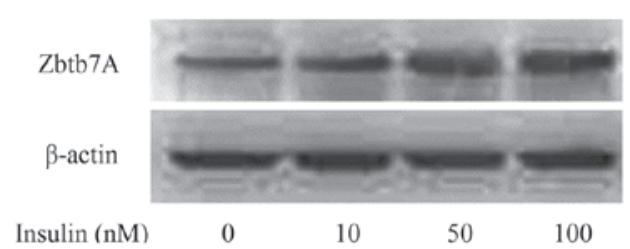

C

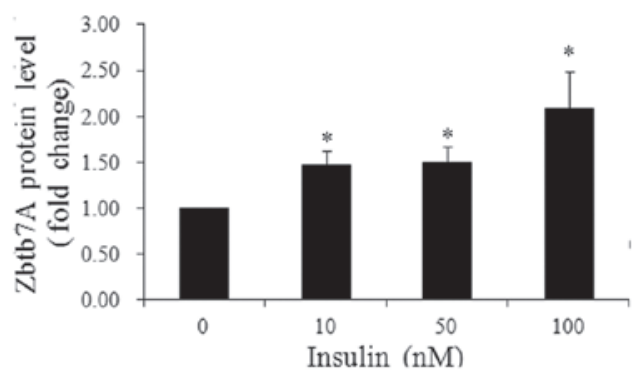

Figure 1. Effect of insulin on Zbtb7A expression. (A) HepG2 cells were cultured with different concentrations of insulin for $24 \mathrm{~h}$. The RNA was extracted and real-time PCR was performed. The Zbtb7A mRNA levels were normalized to GAPDH. Values are expressed as the means $\pm \mathrm{SD}$. ${ }^{*} \mathrm{P}<0.05$ and ${ }^{* *} \mathrm{P}<0.01$ vs. the control group (Zbtb7A mRNA levels in untreated cells, value set as $100 \%$ ). (B) Zbtb7A protein levels were assessed by western blot analysis following treatment of the cells as in (A). (C) The expression of Zbtb7A protein was analyzed by densitometry scanning of the bands and normalized to the Zbtb7A protein band of untreated cells. Values are expressed as the means $\pm \mathrm{SD}$. ${ }^{* *} \mathrm{P}<0.01$ vs. the control group (Zbtb7A protein level in untreated cells, value set as 1).

for $30 \mathrm{~min}$, and then incubated with DMEM containing $100 \mathrm{nM}$ insulin and the indicated inhibitors. It was identified that LY294002 completely blocked the insulin-induced Zbtb7A expression at the mRNA and protein levels, and that PD98059 partially attenuated the effect of insulin on Zbtb7A expression (Fig. 3). The results indicate that insulin enhances Zbtb7A expression predominantly through the PI3K/AKT pathway.

Insulin enhances Zbtb7A promoter activity through Sp1. To elucidate the mechanism of the insulin-induced Zbtb7A expression, wild-type and mutated Zbtb7A promoter-luciferase constructs were developed and transiently transfected into HepG2 cells prior to treatment of the cells with various concentrations of insulin. As shown in Fig. 4A, the activity of the Zbtb7A promoter was increased by insulin in a concentration-dependent manner. Insulin (100 nM) increased Zbtb7A promoter activity by $48.3 \%$ compared with the control group.

Since $\mathrm{Sp1}$ is an effector that mediates the action of insulin on gene expression, we attempted to determine whether $\mathrm{Sp1}$ plays a role in the insulin-induced Zbtb7A expression by
A

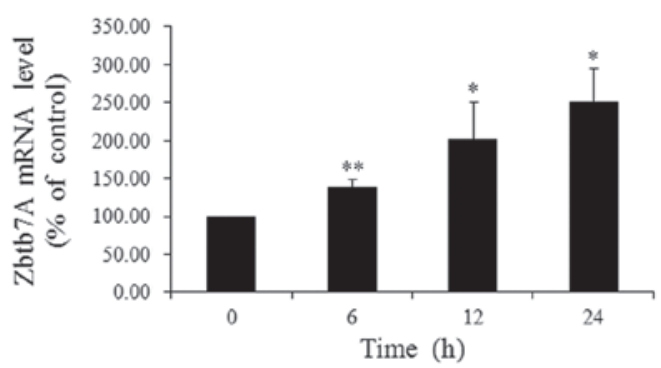

B

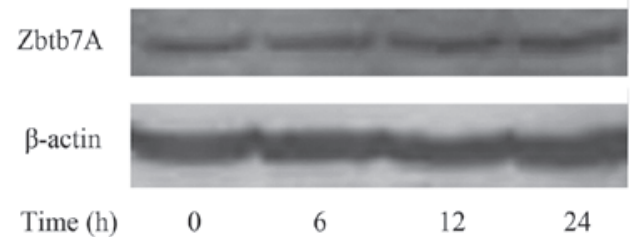

C

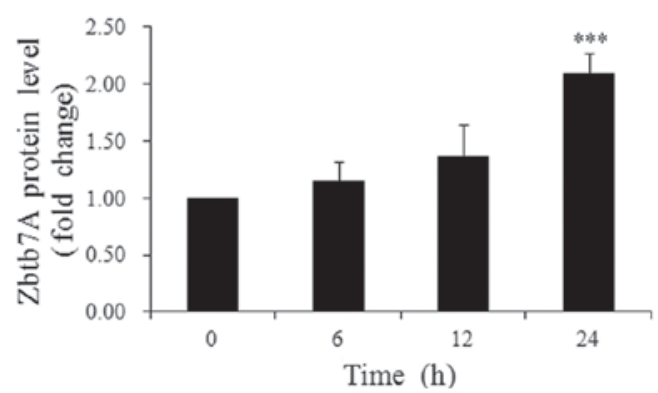

Figure 2. Insulin enhances Zbtb7A mRNA and protein expression in a timedependent manner. (A) HepG2 cells were treated with or without $100 \mathrm{nM}$ insulin for 6,12 or $24 \mathrm{~h}$. The RNA was collected. Zbtb7A mRNA levels were determined by real-time PCR and normalized to GAPDH. Values are expressed as the means $\pm \mathrm{SD}$ for triplicate cultures. ${ }^{*} \mathrm{P}<0.05$ and ${ }^{* *} \mathrm{P}<0.01$ vs. the control group (Zbtb7A mRNA levels in untreated cells, value set as $100 \%$ ). (B) Cells were treated as in (A), and the protein levels of Zbtb7A were analyzed using western blot analysis. (C) The densitometry scans of the bands were normalized to those of the non-insulin-treated cells to obtain the Zbtb7A protein levels. Values are expressed as the means \pm SD. ${ }^{* * *} \mathrm{P}<0.001$ vs. the control group (Zbtb7A protein level in untreated cells, value set as 1).

using a wild-type promoter of Zbtb7A and an Sp1 binding site mutated promoter (Sp1M). Cells transfected with the wild-type or mutated promoters were treated with or without $100 \mathrm{nM}$ insulin for $48 \mathrm{~h}$. As shown in Fig. 4C, the activity of the wild-type promoter was enhanced by insulin, while Sp1M demonstrated no clear response to insulin treatment. Therefore, it may be inferred that insulin enhances Zbtb7A promoter activity through $\mathrm{Spl}$ binding sites.

\section{Discussion}

Our results demonstrate that insulin upregulates Zbtb7A expression in HepG2 cells. To our knowledge, this is the first report that insulin stimulates Zbtb7A promoter activity and enhances endogenous Zbtb7A mRNA and protein levels in a dose- and time-dependent manner, and that the PI3K/AKT cascade and transcription factor $\mathrm{Sp1}$ are responsible for the insulin-induced Zbtb7A expression in HepG2 cells.

To validate the effect of insulin on Zbtb7A expression, we treated HepG2 cells with various concentrations of insulin for $24 \mathrm{~h}$. Cells incubated with $100 \mathrm{nM}$ insulin revealed a 


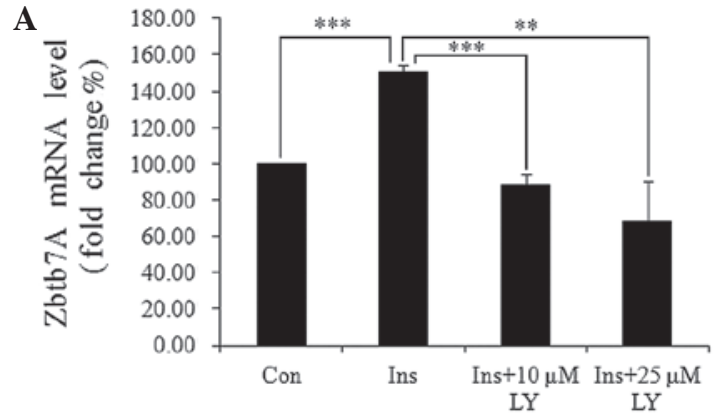

C
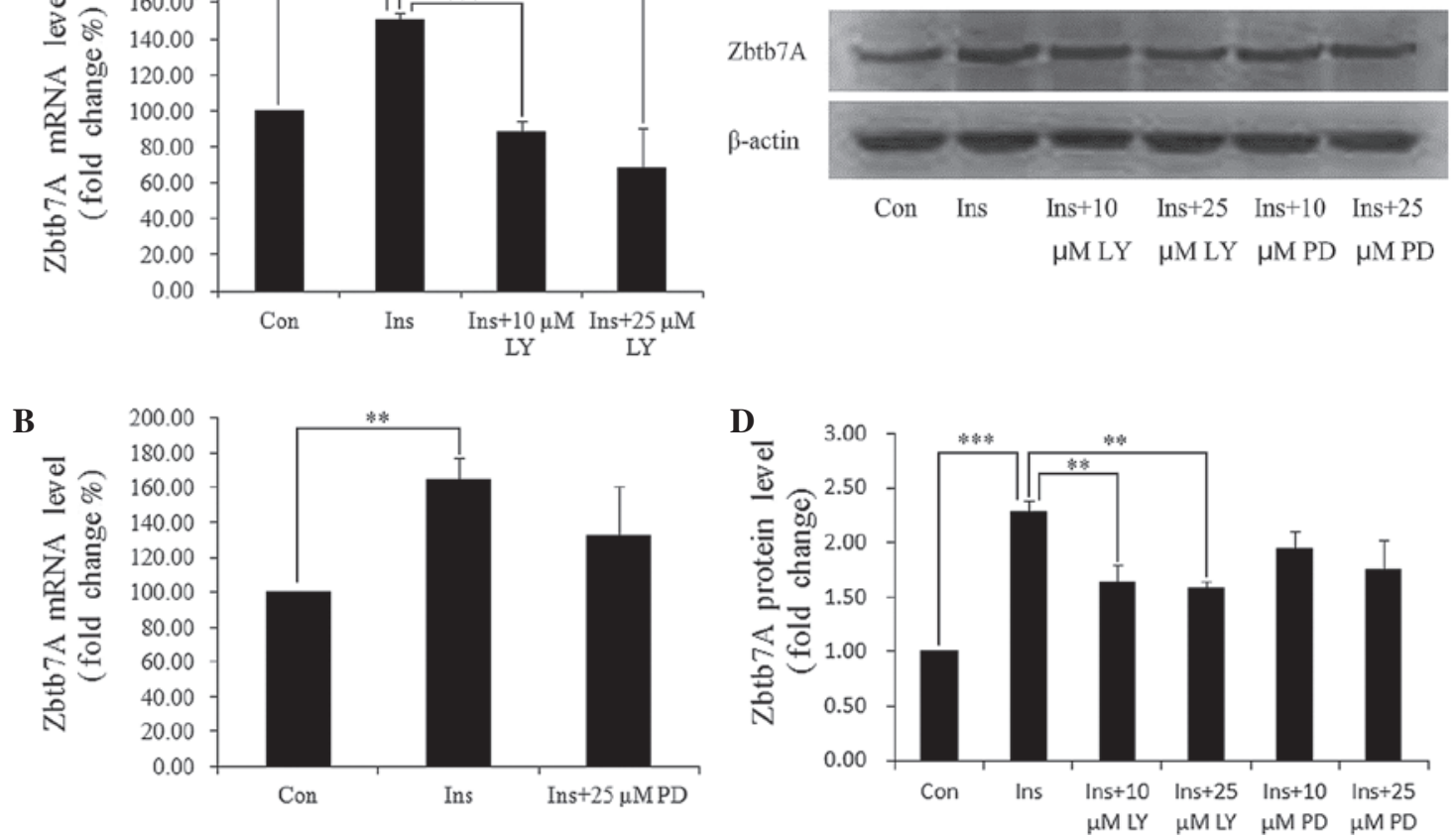

Figure 3. Effects of LY294002 (LY) and PD98059 (PD) on insulin (ins)-induced Zbtb7A expression. Cells were (A) pretreated with 10 or $25 \mu \mathrm{M}$ LY294002 for $30 \mathrm{~min}$, then treated with $100 \mathrm{nM}$ insulin containing 10 or $25 \mu \mathrm{M} \mathrm{LY} 294002$ for $6 \mathrm{~h}$ or (B) pretreated with $25 \mu \mathrm{M}$ PD98059 for 30 min, then treated with $100 \mathrm{nM}$ insulin containing $25 \mu \mathrm{M}$ PD98059 for $6 \mathrm{~h}$. Zbtb7A mRNA levels were analyzed by real-time PCR and normalized to GAPDH. Values are expressed as the means \pm SD for triplicate cultures. ${ }^{* *} \mathrm{P}<0.01$ and ${ }^{* * *} \mathrm{P}<0.001$ vs. the control group (Zbtb7A mRNA levels in untreated cells, value set as $100 \%$ ). $(\mathrm{C})$ Cells were grown in the presence of the indicated inhibitors for $24 \mathrm{~h}$ and the protein levels of Zbtb7A were determined by western blot analysis. (D) The expression of Zbtb7A protein was determined by densitometry scanning of the bands and normalization to the control Zbtb7A protein band level. Values are expressed as the means $\pm \mathrm{SD} .{ }^{* * *} \mathrm{P}<0.01$ and ${ }^{* * *} \mathrm{P}<0.001$ vs. the control group (Zbtb7A protein in untreated cells, value set as 1$)$.
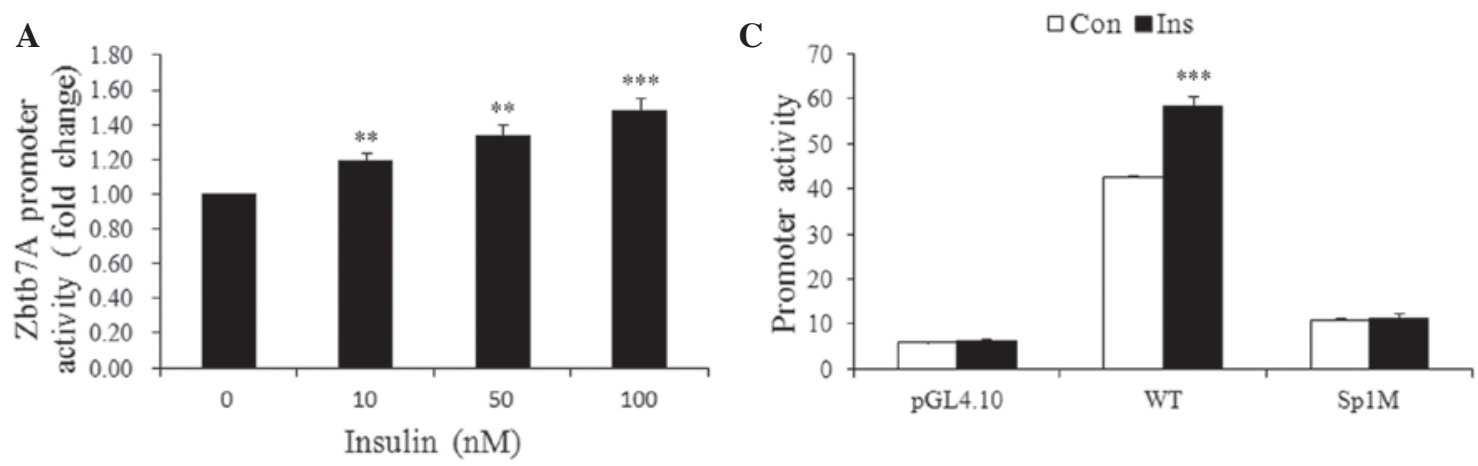

B

$\operatorname{Sp} 1 \mathrm{M}(-641 \sim-636)$

Figure 4. Sp1 is implicated in the insulin (ins)-mediated increase of Zbtb7A promoter activity. (A) HepG2 cells were co-transfected with pLuc1000 promoter luciferase construct and PRLTK plasmid. Twenty-four hours later, the cells were treated with the indicated concentrations of insulin for $24 \mathrm{~h}$. Reporter activity was measured. The promoter luciferase activity was normalized to PRLTK. The data are the means \pm SD of three independent experiments. ${ }^{* *} \mathrm{P}<0.01$ and ${ }^{* * * * *} \mathrm{P}<0.001$ vs. the control (con) group (Zbtb7A promoter activity in untreated cells, value set as 1). (B) The GGGCGG sequence located in -641-636 bp of the Zbtb7A promoter was mutated to TTTTTT. (C) HepG2 cells were transfected with wild-type promoter (WT), mutated promoter (Sp1M), or empty vector pGL4.10 (control), and $24 \mathrm{~h}$ later treated with $100 \mathrm{nM}$ insulin for $24 \mathrm{~h}$. The promoter activities were measured as described above. The data are the means \pm SD of three independent experiments. ${ }^{* * * *} \mathrm{P}<0.001$ compared with the Zbtb7A promoter activity in untreated cells.

maximum 2.17-fold increase in the Zbtb7A mRNA level and a 2.09 -fold increase in the Zbtb7A protein level. We then cultured the cells in DMEM with $100 \mathrm{nM}$ insulin for various times, and tested the mRNA and protein levels of Zbtb7A at the indicated time-points. The highest Zbtb7A protein and mRNA levels were induced by insulin in the HepG2 cells at $24 \mathrm{~h}$. Zbtb7A mRNA was elevated significantly at 6 and $12 \mathrm{~h}$, however, the corresponding protein level showed no evident increase at these times. These differences may be due to the interval between gene transcription and mRNA translation. We used specific inhibitors of different insulin pathways to determine which pathway may be involved in 
the insulin-induced Zbtb7A expression. The observation that LY294002 completely blocked the insulin-induced Zbtb7A expression, while PD98059 attenuated the effect of insulin on Zbtb7A expression to a lesser extent than LY294002, suggests that insulin regulates Zbtb7A expression via the PI3K/AKT pathway. A 1-kb Zbtb7A promoter luciferase construct was developed to identify whether insulin has an effect on Zbtb7A gene promoter activity. The promoter activity was increased 1.48-fold by treatment with $100 \mathrm{nM}$ insulin. However, insulin was not able to boost the activity of a Zbtb7A promoter with an $\mathrm{Sp} 1$ binding site mutation. These results suggest that insulin upregulates Zbtb7A mRNA synthesis through the activation of the Zbtb7A gene promoter via Sp1.

Insulin has the effect of modulating carbohydrate metabolism and regulating certain oncogenes, including c-myc and PTTG (26-29). Messina reported that insulin initially inhibits the proto-oncogene c-myc, but this initial decrease in c-myc is followed by an approximately 3-fold increase in c-myc expression by 60-120 min (27). Thompson and Kakar demonstrated that insulin and IGF-1 stimulate the expression of the oncogene PTTG in a time- and dose-dependent manner through the PI3K/AKT pathway, and they also suggested that insulin-related signaling pathways are a potential molecular target for cancer therapy (26). Since the increased expression of Zbtb7A in non-small cell lung cancer (NSCLC) resulted in carcinogenesis and Zbtb7A had some clinical significance for the prognostic evaluation of patients with NSCLC (30), the stimulation of Zbtb7A by insulin provides a possible mechanism by which insulin contributes to tumor development and/or progression.

Hyperinsulinemia is a common symptom in obese and type 2 diabetic patients. Hepatocellular cancer has increased incidence and prevalence in obesity (31). Compared with the general population, diabetic patients have an increased frequency of hepatitis $\mathrm{C}$, which may contribute to prolonged insulin resistance and liver cancer (32). As a growth factor, insulin is crucial in liver regeneration (33), promotes the growth of the human hepatoma cell line PLC/PRF/5 (34), and reverses the dexamethasone-induced inhibition of rat hepatoma-cell growth and cell-cycle traverse (35). It has been reported that HepG2 cells have intact insulin signaling (36), so we selected HepG2 cells as a cell model to investigate the effects of insulin on Zbtb7A expression. We present evidence of a possible relationship between type 2 diabetes and hepatocarcinoma and our results imply that Zbtb7A might not only be a downstream target of insulin but also an effector of insulin in tumorigenesis.

In conclusion, the present study demonstrates that insulin enhances Zbtb7A expression via activation of the PI3K/AKT cascade and the transcription factor $\mathrm{Sp} 1$ in HepG2 cells. This is the first report concerning the regulation of Zbtb7A expression by insulin, which may have significant implications for the development of diabetes-associated carcinoma and provide insight into the importance of Zbtb7A in clinical treatment.

\section{Acknowledgements}

We thank the financial support from the Ministry of Science and Technology of China (2012ZX09506001-010, 2012CB722605, 2012AA020305 and 2011DFA30620).

\section{References}

1. Maeda T, Hobbs RM, Merghoub T, et al: Role of the proto-oncogene Pokemon in cellular transformation and ARF repression. Nature 433: 278-285, 2005.

2. Morrison DJ, Pendergrast PS, Stavropoulos P, Colmenares SU, Kobayashi R and Hernandez N: FBI-1, a factor that binds to the HIV-1 inducer of short transcripts (IST), is a POZ domain protein. Nucleic Acids Res 27: 1251-1262, 1999.

3. Davies JM, Hawe N, Kabarowski J, et al: Novel BTB/POZ domain zinc-finger protein, LRF, is a potential target of the LAZ-3/BCL-6 oncogene. Oncogene 18: 365-375, 1999.

4. Kukita A, Kukita T, Ouchida M, Maeda H, Yatsuki $\mathrm{H}$ and Kohashi O: Osteoclast-derived zinc finger (OCZF) protein with $\mathrm{POZ}$ domain, a possible transcriptional repressor, is involved in osteoclastogenesis. Blood 94: 1987-1997, 1999.

5. Pessler F, Pendergrast PS and Hernandez N: Purification and characterization of FBI-1, a cellular factor that binds to the human immunodeficiency virus type 1 inducer of short transcripts. Mol Cell Biol 17: 3786-3798, 1997.

6. Apostolopoulou K, Pateras IS, Evangelou K, et al: Gene amplification is a relatively frequent event leading to ZBTB7A (Pokemon) overexpression in non-small cell lung cancer. J Pathol 213: 294-302, 2007.

7. Merghoub T, Cattoretti G, Piazza F, et al: Pokemon is required for cellular differentiation in multiple tissues. Blood 98: 792a, 2001.

8. Maeda T, Hobbs R, Merghoub T, et al: POKEMON is a protooncogene which plays a key role in lymphomagenesis. Blood 104: 951a, 2004.

9. Laudes M, Bilkovski R, Oberhauser F, et al: Transcription factor FBI-1 acts as a dual regulator in adipogenesis by coordinated regulation of cyclin-A and E2F-4. J Mol Med (Berlin) 86: 597-608, 2008.

10. Jeon BN, Yoo JY, Choi WI, Lee CE, Yoon HG and Hur MW: Proto-oncogene FBI-1 (Pokemon/ZBTB7A) represses transcription of the tumor suppressor $\mathrm{Rb}$ gene via binding competition with Sp1 and recruitment of co-repressors. J Biol Chem 283: 33199-33210, 2008.

11. Choi WI, Jeon BN, Yun CO, et al: Proto-oncogene FBI-1 represses transcription of p21CIP1 by inhibition of transcription activation by $\mathrm{p} 53$ and Sp1. J Biol Chem 284: 12633-12644, 2009.

12. Roh HE, Lee MN, Jeon BN, et al: Regulation of Pokemon 1 activity by sumoylation. Cell Physiol Biochem 20: 167-180, 2007.

13. Samson SL and Wong NC: Role of Sp1 in insulin regulation of gene expression. J Mol Endocrinol 29: 265-279, 2002.

14. Zu X, Yu L, Sun Q, et al: SP1 enhances Zbtb7A gene expression via direct binding to GC box in HePG2 cells. BMC Res Notes 2: $175,2009$.

15. Calera MR and Pilch PF: Induction of Akt-2 correlates with differentiation in Sol8 muscle cells. Biochem Biophys Res Commun 251: 835-841, 1998.

16. Roche S, Downward J, Raynal P and Courtneidge SA: A function for phosphatidylinositol 3-kinase beta (p85alpha-p110beta) in fibroblasts during mitogenesis: requirement for insulin- and lysophosphatidic acid-mediated signal transduction. Mol Cell Biol 18: 7119-7129, 1998

17. Rother KI, Imai Y, Caruso M, Beguinot F, Formisano $\mathrm{P}$ and Accili D: Evidence that IRS-2 phosphorylation is required for insulin action in hepatocytes. J Biol Chem 273: 17491-17497, 1998.

18. Slieker LJ, Sloop KW and Surface PL: Differentiation method-dependent expression of leptin in adipocyte cell lines. Biochem Biophys Res Commun 251: 225-229, 1998.

19. Rosen OM: After insulin binds. Science 237: 1452-1458, 1987.

20. O'Brien RM and Granner DK: Regulation of gene expression by insulin. Physiol Rev 76: 1109-1161, 1996.

21. Koontz JW and Iwahashi M: Insulin as a potent, specific growth factor in a rat hepatoma cell line. Science 211: 947-949, 1981.

22. Block GD, Locker J, Bowen WC, et al: Population expansion, clonal growth, and specific differentiation patterns in primary cultures of hepatocytes induced by HGF/SF, EGF and TGF alpha in a chemically defined (HGM) medium. J Cell Biol 132: 1133-1149, 1996.

23. Scassa ME, Guberman AS, Varone CL and Cánepa ET: Phosphatidylinositol 3-kinase and Ras/mitogen-activated protein kinase signaling pathways are required for the regulation of 5 -aminolevulinate synthase gene expression by insulin. Exp Cell Res 271: 201-213, 2001. 
24. Saltiel AR and Pessin JE: Insulin signaling pathways in time and space. Trends Cell Biol 12: 65-71, 2002.

25. Boyd DB: Insulin and cancer. Integr Cancer Ther 2: 315-329, 2003.

26. Thompson AD III and Kakar SS: Insulin and IGF-1 regulate the expression of the pituitary tumor transforming gene (PTTG) in breast tumor cells. FEBS Lett 579: 3195-3200, 2005.

27. Messina JL: Inhibition and stimulation of c-myc gene transcription by insulin in rat hepatoma cells. Insulin alters the intragenic pausing of c-myc transcription. J Biol Chem 266: 17995-18001, 1991.

28. Saltiel AR and Kahn CR: Insulin signalling and the regulation of glucose and lipid metabolism. Nature 414: 799-806, 2001

29. Sun J and Jin T: Both Wnt and mTOR signaling pathways are involved in insulin-stimulated proto-oncogene expression in intestinal cells. Cell Signal 20: 219-229, 2008.

30. Zhao ZH, Wang SF, Yu L, et al: Overexpression of Pokemon in non-small cell lung cancer and foreshowing tumor biological behavior as well as clinical results. Lung Cancer 62: 113-119, 2008.
31. Flegal KM, Carroll MD, Kuczmarski RJ and Johnson CL: Overweight and obesity in the United States: prevalence and trends, 1960-1994. Int J Obes Relat Metab Disord 22: 39-47, 1998.

32. Mason AL, Lau JY, Hoang N, et al: Association of diabetes mellitus and chronic hepatitis $\mathrm{C}$ virus infection. Hepatology 29: 328-333, 1999.

33. Michalopoulos GK and DeFrances MC: Liver regeneration. Science 276: 60-66, 1997.

34. Motoo Y, Kobayashi K and Hattori N: Effect of insulin on the growth of a human hepatoma cell line PLC/PRF/5: a possible role of insulin receptor. Tohoku J Exp Med 156: 351-357, 1988.

35. Spydevold O, Sørensen H, Clausen OP and Gautvik KM: Dexamethasone inhibition of rat hepatoma cell growth and cell cycle traverse is reversed by insulin. Biochim Biophys Acta 1052: 221-228, 1990

36. Au WS, Kung HF and Lin MC: Regulation of microsomal triglyceride transfer protein gene by insulin in HepG2 cells: roles of MAPKerk and MAPKp38. Diabetes 52: 1073-1080, 2003. 